\title{
Relaciones entre Política Económica, análisis del entorno y la motivación del Recurso Humano en las empresas
}

\author{
Rodney Ramírez Arroyo \\ Universidad Fidélitas, rramirez70334@ufide.ac.cr
}

\begin{abstract}
This essay aims to establish how much influence the economic policies do have on staff motivation practices used by business organizations, in the context of their strategic analysis.
\end{abstract}

To achieve this, there will be a tour of theoretical reflections related to the characteristics of the political system and the understanding of economic policies as a product of their interactions.

In a didactic way, specific economic policies are visited considering their objectives and implementation instruments. The link between economic policy and environmental analysis is established; also, the relationship between the inner environmental analysis and human resources management. Finally, a set of company practices to motivate their staff are listed.

All this sequence of concepts, from the political system, economic policy, analysis of the environment and surroundings, the role of human resources and motivational activities; creates a sufficient frame of reference to be able to conclude on the impact of economic policy on the motivation of individuals affiliated to a business system.

Keywords: economy policy, environmental analysis, motivation, human resources..

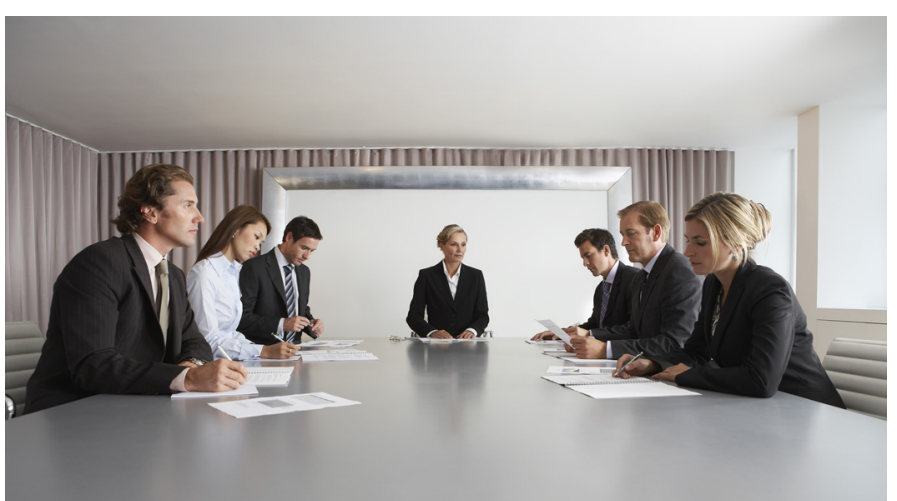

\section{Resumen}

El presente documento pretende establecer desde una perspectiva teórica, el nivel de influencia de las políticas económicas sobre las actividades de motivación del personal utilizadas por las organizaciones empresariales, en el contexto del análisis estratégico de éstas.

Para lograrlo, se hará un recorrido por reflexiones teóricas relacionadas con las características del sistema político y el entendimiento de las políticas económicas como un producto de sus interacciones.

Se visitan de forma didáctica, las políticas económicas específicas en términos de sus objetivos y sus instrumentos de ejecución. Se establece el vínculo entre política económica y análisis del entorno; también la relación entre el análisis del dintorno y la gestión de recursos humanos. Por último, se enumeran un conjunto de prácticas de las empresas para motivar a su personal.

Toda esta secuencia de conceptos, desde el sistema político, la política económica, los análisis del entorno y dintorno, el rol de los recursos humanos y las actividades de motivación; crea el marco de referencia suficiente para poder concluir sobre el impacto de la política económica sobre la motivación de los individuos afiliados a un sistema empresarial.

Palabras clave: política económica, análisis del entorno, motivación, recursos humanos.

\section{Introducción}

Este trabajo busca analizar, desde la perspectiva del análisis estratégico empresarial, los vínculos de la política económica con la gestión de los recursos humanos en las organizaciones empresariales, en particular aquellos que influencian la motivación del personal. La revisión bibliográfica que soporta esta investigación tiene un carácter internacional, por tanto, no es vinculada a un país o región en particular. 
Con tal propósito, se buscará la identificación de los componentes de la política económica, generados desde el sistema político, y su influencia sobre las empresas como entorno y dintorno del mismo sistema.

En la segunda sección, se elucidan los objetivos y los instrumentos de las políticas económicas específicas como lo son: innovación y gestión del conocimiento, comercial, fiscal y monetaria.

Una vez definidas las características de las políticas económicas, se establecerá un enlace entre el análisis de entorno y éstas, y se usará siempre como marco de referencia el sistema político y la importancia de las interacciones entre sus elementos.

La siguiente sección, abordará el análisis del dintorno de la empresa, desde la perspectiva de la gestión del recurso humano. Acá se enfatizará el rol de los individuos en las organizaciones como generadores de influencia y ejecutores de poder.

La quinta parte describe las prácticas y políticas internas usadas por las empresas, desde la administración de los recursos humanos, para promover la motivación entre los miembros de la organización.

La parte seis permite la convergencia de todas las referencias teóricas presentadas en este documento; aprovecha el contexto elaborado para establecer una valoración cualitativa (sustentada en reflexiones teóricas) sobre los vínculos de la política económica con las actividades de motivación utilizadas por las organizaciones empresariales.

\section{La política económica como producto del sistema político}

La comprensión del impacto de la política económica sobre la actividad empresarial no puede limitarse al análisis de los objetivos que esta persigue o los instrumentos que utiliza (Zuñiga, 2016). según apunta Di Gregorio (2007), circunscribir su influencia únicamente a variables como inflación, impuestos o tasas de cambio, ofrece un marco incompleto para que las empresas hagan su análisis estratégico de entorno y por ende se pierde una parte de la efectividad de este. La política económica y la actividad empresarial deben ser racionalizadas en el contexto de las interacciones que se generan en un sistema abierto, al cual ambas pertenecen y donde hay más participantes: el sistema político (Zuñiga, 2016).

Un sistema, según argumenta Easton (1989), es cualquier conjunto de variables que podrían o no estar interrelacionadas entre sí; al elevar esta definición a sistema político, Easton agrega el componente de interacciones. Más específicamente, define al sistema político como un conjunto de interacciones por medio de las cuales se asignan de manera autoritaria valores a la sociedad. En su postura, Easton (1989) observa la sociedad como un conjunto de sus conductas, actitudes e ideas; enumera como ejemplo: economía, cultura, estructura social y los individuos.

El sistema político debe ser comprendido también por su carácter autorreferente (Luhmann, 1997) entendiendo auto referencia como la habilidad de un sistema de producirse y reproducirse por sí mismo en términos de las interacciones de los elementos que los componen. Luhmann destaca que la reproducción del sistema se da por auto contacto entre los elementos de este, descartando el enfoque en que las variables de entradas del sistema sean proveídas únicamente por el ambiente externo. En un sistema autorreferente, las decisiones o acciones tomadas provienen de otras decisiones y de las reacciones de los propios elementos del sistema a cada una de ellas.

La autorreferencia propuesta por Luhmann (1997) se comprende mejor al contrastar las capacidades del sistema político identificadas por Almond y Powell (1996). También aportan al entendimiento de las dinámicas del sistema político, particularmente a través de la capacidad regulativa y la capacidad responsiva del sistema político.

La capacidad regulativa (Almond \& Powell, 1996) se entiende como habilidad del sistema para imponer valores a los otros sistemas de su ambiente; la aceptación o no de los valores impuestos generarán tensiones entre los sistemas que a su vez demandan respuestas de este, estableciéndose un proceso de realimentación e interpretación de entradas y salidas. Acerca de la capacidad responsiva, los mismos autores indican que va más allá de la interpretación de respuestas a la regulación, pues abarca todas las respuestas que el sistema político ofrece a partir de la interpretación de las señales que recibe de los otros elementos del sistema ante cualquier decisión que este genere. Las capacidades responsiva y regulativa del sistema político son precisamente mecanismos de interacción con los que este genera interrelaciones con el entorno; la política económica es un producto de estas (Cuadrado, 2010).

La política económica como conjunto de medidas, decisiones, cursos de acción y objetivos determinados por una autoridad (Cuadrado, 2010), debe entenderse como un producto del sistema político con su carácter abierto, adaptable y autorreferente. Por lo anterior, la política económica como producto o salida del sistema político está en constante realimentación con las interacciones y respuestas de otros elementos del sistema, incluyendo la estructura social y el sistema empresarial.

\section{La política económica, sus objetivos e instrumentos}

Ya entendida la política económica como producto del sistema político, corresponde elaborar sobre su definición, objetivos e instrumentos de ejecución. 
Cuadrado (2010) define la política económica como la aplicación de medidas por parte de las autoridades para el alcance de objetivos específicos. Estos objetivos, según el mismo Cuadrado, están relacionados con el empleo, la estabilidad de precios, el crecimiento económico, la redistribución de las rentas, la calidad de vida y conservación del ambiente. Para el alcance de tales propósitos, la política económica se sustenta en la instrumentalización de políticas específicas: monetaria, comercial y fiscal (Cuadrado, 2010); en adición las contribuciones de Láscaris (2002) y Loray (2017) agregan consideraciones de innovación y gestión de conocimiento para complementar cuatro ejes de acción de la política económica.

Los instrumentos de la política económica inciden de forma evidente sobre las empresas, así lo reconocen Di Gregorio (2007), Loray (2017), Láscaris (2002) y Jiménez (2011). Su influencia general sobre el análisis de entorno empresarial y en específico sobre la motivación del recurso humano será explorada posteriormente en este documento. Antes se hará una breve reseña sobre los objetivos e instrumentos de las políticas económicas.

\section{Innovación y gestión del conocimiento}

Al hablar de innovación, frecuentemente se considera en conjunto a la dimensión científica y tecnológica. Esta es una agrupación razonable si se considera que la innovación está estrechamente vinculada con la gestión del conocimiento, según sugiere Láscaris (2002) y de acuerdo con Schwab (2016) son las principales características de la cuarta revolución industrial.

Según Schwab (2016), la cuarta revolución industrial está distinguida por tecnologías emergentes y la ampliación de la base de conocimiento humano. Los avances tecnológicos y científicos actuales generan niveles de cambio extremadamente veloces, no comparables en lo absoluto con los ritmos observados en las revoluciones industriales previas. Dadas estas condiciones, Schwab remarca la importancia de la regulación y la promoción de políticas específicas para que los países puedan integrarse exitosamente en el flujo de la cuarta revolución industrial.

Loray (2017) ofrece un acercamiento a las políticas específicas de innovación, ciencia y tecnología necesarias para atender las recomendaciones regulatorias de Schwab (2016). Loray (2017), enumera tres categorías para los instrumentos de estas políticas públicas: horizontales, selectivas o sectoriales y focalizadas o de frontera.

Las políticas horizontales, están orientadas a cerrar brechas de conocimiento a través de la formación de capital humano; ofrecen también apoyo a las actividades actuales del sector productivo y proveen incentivos para infraestructura en el sector empresarial (Loray, 2017). Por ejemplo, creación de capacitaciones o el ofrecimiento de financiamientos que apoyen la asimilación de los avances científicos y tecnológicos recientes (Codner \& Del Bello, 2011).
Las políticas selectivas son más específicas en cuanto al direccionamiento de los esfuerzos y requieren de una estructura institucional que soporte su implementación (Codner \& Del Bello, 2011). Apuntan a sectores determinados como de interés por el estado. Incluyen atracción de inversión, promoción de clústeres, incentivos para actividades particulares y programas de apoyo a la competitividad (Loray, 2017).

Por último, las políticas enfocadas se caracterizan por necesitar una articulación mayor entre los diferentes sectores y su ejecución no depende estrictamente del gobierno (Del Bello \& Abeledo, 2007). El modelo de estructura organizacional para innovación tecnológica de Láscaris (2002) enfatiza la importancia de la articulación orgánica entre los sectores investigativo, educativo y productivo. Estas políticas deben tener alcances en los niveles micro, meso, macro y meta de la economía, a la vez que dependen de una estrategia clara y determinada por parte del estado.

\section{Política comercial}

Las políticas comerciales refieren las regulaciones que definen como las empresas y los individuos conducen negociones con partes de otros países. Define las estrategias clave en términos de exportación, importación y términos de intercambio (Lukaukas, Stern, \& Zanini, 2013).

Lakaukas, Stern y Zanini (2013) identifican entre los objetivos de la política comercial: el incremento y la diversificación de las exportaciones, protección a sectores productivos incipientes, mejoramiento de los términos de los intercambios comerciales, estabilidad del tipo de cambio.

En el mismo documento se listan los principales instrumentos de la política comercial: los aranceles, los sistemas de cuotas, control cambiario (instrumento también asignable a la política monetaria) y los subsidios.

En relación con los aranceles, Fuders (2014) amplía que las barreras al comercio trascienden la mera aplicación de barreras o aperturas arancelarias. Identifica la existencia de barreras paraarancelarias, explicándolas como tributos que tienen el mismo efecto que los aranceles, por ejemplo, restricciones de importación, regulación de tránsito o incluso controles de frontera.

\section{Política fiscal}

Según la Enciclopedia Británica (2019), la política fiscal tiene como propósito primordial el resguardo gubernamental de los gastos y los impuestos. Está directamente relacionada con las finanzas del estado, pues se resume en control de gastos e ingresos. Se ejecuta a través de los niveles de gasto del gobierno y la fijación y recolección de cargas tributarias. 
En términos de objetivos, diversas fuentes hacen un paralelismo entre la política monetaria y la fiscal (Cuadrado, 2010; De Gregorio, 2007; Martner, 2000). Las coincidencias entre ellos señalan el control del desempleo, crecimiento económico, estabilización de precios y salarios.

Del mismo modo, la literatura consultada (Cuadrado, 2010; De Gregorio, 2007; Martner, 2000) concuerda acerca de la instrumentación disponible para la política fiscal: la fijación de cargas tributarias y el control del gasto público. De modo muy general, se establece que los impuestos regulan la cantidad de dinero disponible para el consumo mientras se puede promover el gasto del gobierno; adicionalmente tienen un efecto sobre la cantidad de inversión extranjera que arriba a un país. En cuando a los ajustes en gastos del gobierno, su incremento es percibido como un estímulo a la actividad económica, que hace dinero disponible en la economía aumentando el consumo y posiblemente disminuyendo el desempleo. Sin excepción, las fuentes convergen en un delicado equilibrio en el uso de la instrumentación para lograr los efectos deseados y en el estrecho vínculo interrelacionado entre la política fiscal y la monetaria.

\section{Política monetaria}

La lectura de Martner (2000) y De Gregorio (2007) permite inferir que la política monetaria es el conjunto de medidas de la política económica, determinadas por los bancos centrales, para influenciar los niveles de actividad en la economía. Generalmente se vale del control de la disponibilidad de dinero en la economía, el tipo de cambio y la administración de las tasas de interés.

Tanto Cuadrado (2010), De Gregorio (2007) y Martner (2000) destacan los objetivos comunes de las políticas monetarias y fiscales. Como se mencionó en el apartado anterior entre ellos se cuentan: control del desempleo, crecimiento económico, estabilización de precios y salarios. En particular tanto Martner como Cuadrado, enfatizan que la modulación de las metas inflacionarias actúa como punto de convergencia y corrección de las acciones para el alcance de los demás objetivos de la política monetaria.

Desde una perspectiva genérica, los conjuntos de medidas de la política monetaria pueden clasificarse en dos tipos: de expansión y contracción. La primera relacionada con bajas tasas de interés para promover la inversión, el consumo, la disponibilidad de dinero y la generación de empleo. La segunda actúa a la inversa en relación con los intereses y el dinero disponible, pero se logra un efecto de reducción de la inflación (Encyclopædia Britannica, 2019).

En términos de la instrumentación usada, los bancos centrales cuentan con prácticas como el endeudamiento a través de operaciones de mercado abierto (para aumentar la cantidad de dinero en el sistema), manejo de la tasa de política monetaria (la tasa a la que otros bancos pueden recibir recursos del banco central) y variaciones en los porcentajes de reserva obligatorios, conocido también como encaje bancario (Cuadrado, 2010).

\section{Análisis del entorno empresarial y política económica}

Ya planteado el carácter abierto, adaptable y autorreferente del sistema político y establecido que la política económica es un producto de este sistema; resulta evidente el incluir al sistema empresarial en el contexto de las interrelaciones generadas en el sistema político. Como elemento relevante en el sistema político, las empresas están sujetas a la asignación autoritaria de medidas, así como a la valoración obligatoria de estas, a la determinación de su reacción propia y a la observación de las reacciones de otros elementos en el sistema. En resumen, la empresa está inmersa en un ambiente complejo, evolutivo, de lógica circular y en constante retroalimentación (Zuñiga, 2016).

Zuñiga (2016) anota que los métodos clásicos de análisis estratégico empresarial se han limitado a la anticipación de relaciones bilaterales con proveedores, clientes, entidades de gobierno, accionistas, las comunidades y otras partes interesadas. La dimensión política del análisis del entorno se ha restringido a una cuantificación estática de las regulaciones gubernamentales. Este tipo de análisis coarta la capacidad de las empresas de entender los rasgos fundamentales del entorno, que como indica Zuñiga es turbulento, relacional, multidimensional y global.

Esta privación en la compresión de la complejidad del ambiente, confina la toma de decisiones estratégicas en las empresas a la valoración de interacciones binarias y en la mayoría de los casos unidireccionales. Como señala Shwaninger (2000), el camino hacia una organización inteligente lleva consigo la adquisición de habilidades particulares como: adaptación a condiciones cambiantes, la capacidad para influenciar el ambiente, encontrar nuevos giros de negocio si fuera necesario e incluso poder contribuir a la sostenibilidad del entorno como un todo.

En una referencia directa hacia las interrelaciones de la política económica con el sistema empresarial, Martner (2000) va más allá de las circunspecciones teóricas de las políticas económicas y añade elaboraciones relacionadas con las interacciones de los diferentes participantes del sistema político: establece la complejidad dogmática y pragmática del sistema.

En un análisis del entorno referido a política económica, las consideraciones fiscales y monetarias, por ejemplo, no pueden verse por separado (De Gregorio, 2007). Es importante comprender el resultado de las interacciones entre ambas: las tasas de interés, el tipo de cambio, la carga tributaria, el control de precios, los estímulos gubernamentales, la disponibilidad de dinero. Por ejemplo, una política fiscal expansiva puede derivar en déficit fiscal, que a su vez podría disparar una colocación excesiva de dinero en el sistema para financiar el desbalance; esto 
sube las tensiones inflacionarias y posiblemente impacte las tasas de interés con implicaciones en el tipo de cambio (Martner, 2000).

Los aportes más notables de Martner (2000), no están precisamente en el esbozo teórico de ambas políticas; están en las consideraciones relacionales que establece. Plantea una fuerte injerencia política sobre las políticas económicas; según su tesis no es tan relevante la selección y uso de algún instrumento en especial, más bien es determinante como el sistema reacciona a los estímulos ambientales y a los resultados inmediatos de su gestión: crecimiento económico, brecha del PIB, inflación o desempleo. Martner deja inquietudes que no se deben omitir en un análisis del entorno empresarial. Por ejemplo, los planes de los bancos centrales o gobiernos pueden estar claros y parecer pertinentes a las necesidades de un país, pero ¿cómo reaccionan el gobierno y los banqueros a las presiones del entorno?, ¿cuál es la credibilidad del sistema político en relación con el cumplimiento de su planificación?, o bien ¿cuál es la consistencia ejecutiva de los entes responsables? La lectura de Martner refuerza la importancia de la compresión de las interrelaciones entre quienes actúan en el sistema político y de entender la política económica como un producto de todas las interacciones dentro del sistema.

Un análisis estratégico del entorno debe ubicar a la empresa en el contexto del sistema político; entender su rol adaptable, influenciador y contributivo; abrazar la complejidad y darle un sentido que soporte una buena toma de decisiones. Sin embargo, el análisis del entorno no es todo lo que una buena estrategia necesita, se debe añadir la perspectiva interna, eso se discute a continuación.

\section{Análisis del dintorno y la gestión de los recursos humanos}

Esta sección tiene como propósito definir el rol de la administración de los recursos humanos en el análisis estratégico empresarial. Para tal fin se elabora sobre la influencia de los recursos humanos en la actividad empresarial, acerca de las características del análisis del dintorno y del rol del individuo en la empresa y como participante también del sistema político.

La administración de los recursos humanos ha ganado un lugar relevante en los análisis estratégicos de las empresas (Rothwell \& Kazanas, 2003). Como su nombre lo sugiere, esta disciplina está íntimamente vinculada con la gestión de las personas que componen una organización. Rothwell y Kazanas (2003) establecen que la administración de los recursos humanos provee un enfoque estratégico instrumentalizado a través de prácticas y políticas internas que regulan las relaciones laborales.

Chitescu y Lixandru (2015) han determinado una influencia de los sistemas sociales, políticos y económicos sobre los recursos humanos. Ochetan y Ochetan (2012) también elaboraron sobre esta línea agregando el impacto de las crisis financieras sobre las personas como integrantes de una organización empresarial. Cania (2017), relacionó la efectividad de las prácticas y políticas internas de la administración de los recursos humanos con el desempeño de la organización. Mintzberg (2000) destacó la influencia de los miembros de una organización sobre esta, desde una perspectiva de relaciones y ejercicio del poder.

Lo anterior refuerza que en el contexto del sistema político, todas las partes, incluso aquellas participando en subsistemas, están expuestas a las interacciones y respuestas del sistema político. Para el caso particular de la administración de los recursos humanos, en su rol de subsistema dentro de un sistema empresarial, se puede concluir que genera consideraciones relevantes en el análisis estratégico empresarial, en especial desde una perspectiva interna que es afectada por factores externos e internos de la organización.

Las reflexiones sobre los procesos internos de las organizaciones son parte fundamental del análisis estratégico y sus considerandos son distintos a los del análisis de entorno. Zuñiga (2016) en su planteo sobre el análisis del dintorno, lo identifica como complementario al análisis del entorno y lo enmarca principalmente en función de las interacciones de los individuos dentro de la empresa.

Zuñiga (2016) observa el dintorno organizacional como una escalación del carácter autorreferente y adaptable del sistema político al microcosmos de una organización, reconociendo su complejidad intrínseca. Perfila una propuesta de análisis de dintorno fundamentada en una perspectiva relacional, en las dinámicas de poder y en la posibilidad de influencia de los individuos en la empresa.

Expuesto lo anterior se concluye que la administración del recurso humano como conjunto de prácticas y políticas internas que norman las relaciones humanas en una organización es una de las consideraciones relevantes en el análisis del dintorno y por tanto parte del análisis estratégico de las empresas.

\section{Gestión de la motivación del recurso humano}

Este apartado tiene como propósito, conocer las prácticas y políticas de la administración de los recursos humanos relacionadas con la motivación del personal en la empresa. En párrafos anteriores se definió la importancia de la gestión de los recursos humanos en el análisis estratégico de la organización, en particular en la vertiente del dintorno. Ahora mediante el análisis de objetivos e instrumentos, se caracterizarán las prácticas que usan las empresas para mantener la motivación en su fuerza laboral.

La motivación es usualmente entendida como un fenómeno estrictamente personal (Osabiya, 2015), sin embargo, el individuo en el contexto empresarial y como parte del 
sistema político tiene influencia y la posibilidad de ejercer poder sobre la organización (Mintzberg, 1982); por tanto, el estado motivacional de un grupo de personas es relevante para las empresas y su análisis estratégico.

Según Ritter y Taylor (1997), el alcance de los objetivos estratégicos de una organización se da a través de los individuos y sus comportamientos; la gestión de recursos humanos, mediante sus prácticas y políticas busca influenciar los comportamientos de las personas en favor del alineamiento con los objetivos estratégicos de la empresa. Este enunciado termina de vincular la importancia de la motivación del recurso humano en los análisis estratégicos de las empresas.

En la siguiente sección, se relacionará la instrumentalización de la política económica con las acciones y políticas internas de la administración de recursos humanos orientadas a mantener la motivación del personal de la empresa. Antes de llegar ahí, se tomará la propuesta de Boswell, Colvin y Darnold (2008) para describir puntualmente las actividades empresariales tendientes a motivar a las personas de la organización.

Cultura y clima organizacional. Se enfoca en como los miembros de la empresa, de forma compartida, experimentan y entienden a la organización. Por un lado, el clima refiere percepción del grupo de individuos, por otro la cultura apunta a los supuestos y a la ideología generalizada. Ambos persiguen que las personas tengan aceptación social, entendimiento de las reglas de desempeño y alineamiento estratégico al proveer una expectativa clara de los comportamientos esperados por la organización.

Sistemas de compensación y beneficios. Sirven como el reconocimiento primario en retorno por un comportamiento alineado con las exceptivas de la empresa. Es la más tangible de las medidas de la administración para promover comportamientos motivado y alineados con las metas establecidas.

Gestión del desempeño. Consiste en la medida, valoración y retroalimentación del desempeño de la persona. Es una señal clara de que la persona está alineada con las necesidades de la empresa. Le ofrecen al individuo la certeza de entender lo que se espera de su comportamiento y de recibir señales de corrección oportunas, también enviste a la relación persona-empresa de una condición de justicia.

Desarrollo de carrera. Plantea una expectativa de progreso para las personas. Produce una percepción positiva en el individuo en términos de su relación con la entidad. Desarrolla de forma tangible las habilidades y competencias de la persona. Busca incrementar el alineamiento con los objetivos estratégicos y mejorar la base de conocimiento empresarial.
Seguridad del empleo. La percepción de una relación inestable entre el individuo y la empresa crea desmotivación en las personas. Si bien la interpretación de seguridad laboral no incrementa la motivación por sí misma, la falta de esta tiene un efecto negativo sobre la motivación del personal.

Involucramiento del individuo. Ofrece a la persona sentirse parte de los procesos de toma de decisiones en la organización. La sensación de relevancia, aprecio y respeto por una opinión tiene un efecto favorable en la motivación.

Administración por valores y motivación intrínseca. Logra en el personal un sentido de propósito donde los individuos encuentran satisfacción en sus tareas al margen de las recompensas y beneficios que la organización ofrezca. La administración por valores genuina obtiene un nivel de involucramiento y compromiso que asegura alineamiento con los objetivos estratégicos de la empresa.

\section{Efecto de la política económica sobre la motivación del recurso humano}

Llegado este punto, se obtiene la convergencia de todo el esbozo teórico que permite la determinación de la influencia de las políticas económicas sobre la motivación del personal en el marco del análisis del entorno de las empresas.

Hasta ahora se ha considerado el carácter adaptable, autorreferente y complejo de las interacciones de todos los participantes del sistema político; la política económica como producto en realimentación constante con el sistema político; las políticas económicas específicas en términos de sus objetivos y sus instrumentos de ejecución; se estableció el vínculo entre política económica y análisis del entorno; también la relación entre el análisis del dintorno y la gestión de recursos humanos; por último se enumeraron un conjunto de prácticas de las empresas para motivar a su personal.

Toda la secuencia de relaciones mencionada en el párrafo anterior ha tendido el puente para establecer la influencia de la política económica sobre la motivación de los individuos afiliados a un sistema empresarial.

Para determinar la influencia de las políticas económicas sobre la motivación, se presentará una matriz (ver Figura 1) con las principales instrumentaciones de las políticas económicas en par con las prácticas y políticas internas de la gestión de los recursos humanos en procura de la motivación del personal.

Como se observa en la Figura 1, en total se tienen siete prácticas de motivación que serán ubicadas como columnas y doce instrumentos de política económica que se fijarán como filas. Para cada intersección "práctica motivación / instrumento de política económica”, se establece una escala 
discreta de valores entre 1 y 3 . Adicionalmente, al valor numérico se asigna un código de color que permita observar con mayor facilidad la concentración de las incidencias de interés. Importante acotar que las calificaciones en las intersecciones de la matriz serán asignadas por el autor que escribe, basado en la revisión bibliográfica que permitió la elaboración de este artículo.

El valor 1, estará asociado con el color verde e indica una injerencia débil del instrumento económico con la práctica de motivación. Por ejemplo, las políticas de investigación y gestión de conocimiento influencian en poca medida la administración de valores, esto por cuanto el conocimiento tecnológico no está enfocado en los valores personales o empresariales.

El valor 2, asociado con el color amarillo representa una interacción media entre la política económica y la práctica de motivación. Para ilustrar considérese que las políticas fiscales y monetarias tiene un impacto medio sobre la motivación por desarrollo de carrera, dado que los presupuestos de capacitación en las empresas pueden verse reducidos según actúe el gobierno y los bancos centrales.

El valor 3, ligado al color rojo indica una fuerte influencia de la política económica sobre la práctica de motivación. Tal es el caso del control cambiario sobre la seguridad en el empleo: dependiendo de la dirección del cambio y del giro del negocio (importación o exportación) podría haber una contracción en la actividad que genere despidos.

En general, pueden extraerse las siguientes conclusiones de la observación de la Figura 1:

- El involucramiento del individuo y la administración por valores, en general son poco influenciados por la política económica.

- El desarrollo de carrera es fuertemente influenciado por las políticas de innovación y gestión del conocimiento.

- La política monetaria y la fiscal inciden con intensidad sobre los sistemas de compensación y la seguridad del empleo.

- Salvo por las políticas de control cambiario, la política comercial no influye con fortaleza a las prácticas de motivación.

- La gestión del desempeño y la cultura organizacional son modernamente afectadas por las políticas económicas en general.

Si bien el mapa de calor muestra diversidad en los niveles de influencia de la política económica sobre las prácticas de motivación, más de la mitad (65\%) de las interacciones se valoraron como de media y alta influencia; un $17 \%$ se marcaron de incidencia fuerte; todas las políticas económicas tienen al menos una actividad de motivación sobre la que influyen fuertemente.

Figura 1. Matriz de influencia entre políticas económicas y prácticas de motivación del personal de las empresas

\begin{tabular}{|c|c|c|c|c|c|c|c|c|}
\hline \multicolumn{2}{|c|}{$\begin{array}{l}\text { Políticas } \\
\text { económicas }\end{array}$} & \multirow{2}{*}{$\begin{array}{c}\begin{array}{c}\text { Cultura y } \\
\text { Clima } \\
\text { Organizacional }\end{array} \\
2\end{array}$} & \multirow{2}{*}{$\begin{array}{c}\begin{array}{c}\text { Sistemas de } \\
\text { compensación } \\
\text { y beneficios }\end{array} \\
2\end{array}$} & \multirow{2}{*}{$\begin{array}{c}\begin{array}{c}\text { Gestión del } \\
\text { desempeño }\end{array} \\
2\end{array}$} & \multirow{2}{*}{$\begin{array}{c}\begin{array}{r}\text { Desarrollo } \\
\text { de carrera }\end{array} \\
3\end{array}$} & \multirow{2}{*}{\begin{tabular}{|c}
$\begin{array}{r}\text { Seguridad } \\
\text { del empleo }\end{array}$ \\
1 \\
\end{tabular}} & \multirow{2}{*}{\begin{tabular}{|c|}
$\begin{array}{c}\text { Involucramiento } \\
\text { del individuo }\end{array}$ \\
1 \\
\end{tabular}} & \multirow{2}{*}{$\begin{array}{c}\begin{array}{c}\text { Administración } \\
\text { por valores }\end{array} \\
1\end{array}$} \\
\hline Innovación y & Políticas horizontales & & & & & & & \\
\hline gestión de & Políticas selectivas & 2 & 2 & 2 & 3 & 1 & 1 & 1 \\
\hline conocimiento & Políticas enfocadas & 2 & 2 & 2 & 3 & 1 & 1 & 1 \\
\hline \multirow{4}{*}{$\begin{array}{l}\text { Política } \\
\text { comercial }\end{array}$} & Barreras arancelarias & 1 & 2 & 1 & 1 & 2 & 1 & 1 \\
\hline & Sistemas de cuotas & 1 & 2 & 1 & 1 & 1 & 1 & 1 \\
\hline & Control cambiario & 2 & 3 & 1 & 1 & 3 & 1 & 1 \\
\hline & Subsidios & 1 & 2 & 1 & 1 & 2 & 1 & 1 \\
\hline \multirow{2}{*}{$\begin{array}{l}\text { Política } \\
\text { fiscal }\end{array}$} & $\begin{array}{l}\text { Fijación de cargas } \\
\text { tributarias }\end{array}$ & 2 & 3 & 2 & 2 & 3 & 1 & 1 \\
\hline & $\begin{array}{l}\text { Determinación del } \\
\text { gasto público }\end{array}$ & 2 & 3 & 2 & 2 & 3 & 1 & 1 \\
\hline \multirow{3}{*}{$\begin{array}{c}\text { Política } \\
\text { monetaria }\end{array}$} & $\begin{array}{l}\text { Operaciones } \\
\text { de mercado }\end{array}$ & 2 & 3 & 2 & 2 & 3 & 1 & 1 \\
\hline & $\begin{array}{l}\text { Tasa de política } \\
\text { moneria }\end{array}$ & 2 & 3 & 2 & 2 & 3 & 1 & 1 \\
\hline & Encaje bancario & 2 & 3 & 2 & 2 & 3 & 1 & 1 \\
\hline
\end{tabular}

Notas: 1-Verde: baja influecia; 2-Amarillo: influencia moderada; 3-Rojo influecia fuerte.

Las valoraciones son asigandas por el autor sobre la base bibliográfica investigada.

Fuente: Elaboración propia, 2020 


\section{Conclusiones}

El recorrido bibliográfico realizado en este trabajo permitió responder la pregunta planteada en la introducción: ¿Cuáles son los vínculos, desde la perspectiva del análisis estratégico en las empresas, de la política económica con la gestión gerencial de los recursos humanos en estas, en términos de motivación?

Con base en revisión bibliográfica y el análisis reflexivo de carácter conceptual, se concluye que la política económica tiene un impacto sobre las prácticas de motivación en las organizaciones. Al ser esta una investigación teórica, se subraya la necesidad de abundantes estudios empíricos, para contrastar la conclusión que acá señalada.

Fue posible identificar influencias de variada intensidad según el tipo de política económica y la clase de actividad motivacional con la que cuentan empresas. Destaca la fuerte incidencia de las políticas monetarias, fiscales y de control cambiario sobre compensación y beneficios y la seguridad del empleo. También con influencia marcada se encontró a las políticas de innovación y gestión del conocimiento en relación con el desarrollo de carrera que las empresas ofrecen a su personal.

Se identificó que no todas las prácticas de motivación se ven fuertemente influenciadas por alguna política económica, tal es el caso de la administración por valores y el involucramiento del individuo. La revisión bibliográfica realizada no alcanza para descartar las influencias sobre las mencionadas prácticas de motivación; sin embargo, tampoco ofrece pistas sobre la vulnerabilidad de estas actividades a las políticas económicas. Cabe destacar que el carácter abierto, adaptable y autorreferente del sistema político en la que se han enmarcado todas las reflexiones teóricas de esta investigación, abre espacio para que puedan existir impactos e influencias que no resulten evidentes ante un primer acercamiento teórico.

La investigación también permitió identificar a la política económica como un producto de las interacciones generados a partir del sistema político en su carácter abierto, adaptable y autorreferente. La premisa anterior permite ampliar las consideraciones de política económica en el análisis estratégico del entorno en las empresas; los hallazgos retan a quien analiza a trascender los meros postulados teóricos clásicos sobre economía y política y a recurrir a la valoración de las interacciones, las reacciones $\mathrm{y}$ las realimentaciones que proveen quienes participan inmersos en el sistema político.

Un último producto de este documento está referido al rol de la administración de los recursos humanos en el análisis del dintorno empresarial. La consulta bibliográfica derivó en la relevancia de las relaciones entre individuos en términos de influencia y ejercicio del poder para entender y tomar decisiones relativas al dintorno de la organización. Y dado que la interacción entre individuos está determinada por el comportamiento de estos, las actividades y políticas internas para la motivación generadas desde la gestión de los recursos humanos, se vuelven insumos importantes para la modificación de conductas individuales y grupales a lo interno de la organización.

\section{Referencias}

Almond, G., \& Powell, G. (1996). Comparative politics. Washington: L, B \& Co.

Boswell, W., Colvin, A., \& Darnold, T. (2008). Organizational Systems and Employee Motivation. (R. Kanfer, G. Chen, $\&$ R. Pritchard, Eds.) Work motivation: Past, present, and future, 361-400.

Cania, L. (2017). The Impact of Strategic Human Resource Management on Organizational Performance.

Chitescu, R., \& Lixandru, M. (2016, 12). The Influence of the Social, Political and Economic Impact on Human Resources, as a Determinant Factor of Sustainable Development. Procedia Economics and Finance, 39, 820826. doi:10.1016/S2212-5671(16)30259-3

Codner, D., \& Del Bello, J. C. (2011). VI Congreso Internacional de Sistemas de Innovación para la Competitividad. México.

Cuadrado, J. (2010). Política económica. Elaboración, objetivos e instrumentos. Madrid: Mc. Graw Hill.

De Gregorio, J. (2007). Economía internacional. Claves teóricoprácticas sobre la inserción de Latinoamérica en el mundo. Santiago: Pearson Education.

Del Bello, J. C., \& Abeledo, C. (2007). Reflexiones sobre cuestiones pendientes de la Agenda de Política en Ciencia, Tecnología e Innovación de Argentina.

Easton, D. (1989). Diez textos básicos de Ciencia Política. (A. Batlle, Ed.) Barcelona: Arial.

Encyclopædia Britannica. (30 de 08 de 2019). Fiscal policy. (T. E. Britannica, Editor) Recuperado el 31 de 10 de 2020, de Encyclopædia Britannica: https://www.britannica.com/ topic/fiscal-policy

Funders, F. (2007). Las herramientas de la política comercial: barreras arancelarias y para arancelarias. Economía Internacional. Claves teórica-prácticas sobre la inserción de Latinoamérica en el Mundo, 63-68.

Furuoka, F. (2015). Human resources, physical resources and economic development: A foundation of human resource economics. Recuperado el 31 de 10 de 2020, de University Library of Munich: https://mpra.ub.uni-muenchen. de/61666/

Hanno, M. (2017, 4). The Impact of Strategic Human Resource Management on Organizational Performance. The Impact of Strategic Human Resource Management on Organizational Performance. 
Jimenez, F. (2011). Crecimiento económico. Enfoques y modelos. Lima: Fondo Editorial/Pontificia Universidad Católica del Perú.

Katz, H. C., \& Colvin, A. J. (2008). Organizational systems and employee motivation[Electronic version]. Recuperado el 30 de 10 de 2020, de Cornell University, ILR School site: https://digitalcommons.ilr.cornell.edu/articles/1313

Láscaris, T. (2002). Estructura organizacional para la innovación tecnológica. El caso de América Latina. Revista Iberoamericana de Ciencia, Tecnología e Innovación(3).

Loray, R. (2017). Políticas públicas en ciencia, tecnología e innovación: tendencias regionales $\mathrm{y}$ espacios de convergencia. Revista de Estudios Sociales(62).

Luhmann, N. (1997). Teoría Política en el Estado de Bienestar. Madrid: Alianza Universidad.

Lukaukas, A., Stern, R., \& Zanini, G. (2013). Handbook of trade policy for development. Oxford: Oxford Scolarship Online.

Martner. (2000). Estrategias de política económica en un mundo incierto. Santiago: Naciones Unidas / Instituto Latinoamericano y el Caribe de Planificación Económica y Social.

McRee, C. (2018). Managerial perceptions of employee motivation. Theses and Dissertations, 1001.

Mintzberg, H. (1982). Power in and around organizations. Englewoodds Cliffs, N.J.: Prentice-Hall Inc.

Mirela, M. (2015, 9). CORRELATION BETWEEN HUMAN NEEDS SYSTEM - PERSONALITY - HUMAN MOTIVATION. Ecoforum, 4.

Mitxeo, J., \& Otros. (2004). Los clústeres como fuente de competitividad: el caso de la comunidad de la Comunidad Autónoma del País Vasco. Cuadernos de gestión, 4(1).

Ochetan, C., \& Ochetan, D. (2012, 12). The Influence of Economic and Financial Crisis on Human Resources Management. Procedia Economics and Finance, 3, 769774. doi:10.1016/S2212-5671(12)00228-6
Ojogwu, A. (2013, 1). THE ENVIRONMENT OF HUMAN RESOURCE MANAGEMENT: A CHALLENGE TO SUSTAINABLE DEVELOPMENT IN NIGERIA. Kogi Journal of Management, 5.

Osabiya, B. $(2015,5)$. The effect of employees motivation on organizational performance. Journal of Public Administration and Policy Research, 7, 62-75. doi:10.5897/ JPAPR2014.0300

Peng, M. (2010). Estrategia global. Querétaro: Cengage Learning.

Ritter, J., \& Taylor, L. (1997, 2). Economic Models of Employee Motivation. Review, 79, 3-21. doi:10.20955/r.79.3-22

Rothwell, W., \& Kazanas, H. (2003). Planning And Managing Human Resources. Amherst, Mass.: HRD Press.

Schwab, K. (2016). The Fourth Industrial Revolution. Cologny/ Geneva: World Economic Forum.

Shwaninger, M. (2000). Managing Complexity. The Path Toward Intelligent Organizations. Systemic Practice and Action Research, 13(2).

Sinisterra, M. (2007). Desfragmentación de la producción y Multilocalización. La ventaja comparativa reconsiderada. Economía internacional. Claves teórico-prácticas sobre la inserción de Latinoamérica en el mundo, 127-147.

Stefanescu, F., \& Dărăbăneanu, D. (2011, 1). THE HUMAN RESOURCES MANAGEMENT IN THE CONTEXT OF THE ECONOMIC CRISIS. Romanian Economic Business Review, 6, 104-117.

Zuñiga, C. (2016). Gerencia Política: organización, poder y toma de decisiones estratégicas. Tesis de grado para optar por el grado de doctor en Ciencias de la Administración. San José: UNED. 